\title{
Devant cet impuberisme quels sont vos diagnostics?
}

\author{
Walid DENGUEZLI, Zina CHOUKET, Raja FALEH, Anis HADDAD, \\ Zahra SAIDANI, Hayet LAAJILI, Mohamed SAKOUHI
}

Service de Gynécologie Obstétrique, CHU Fattouma Bourguiba, Monastir, Tunisie

\section{RESUME}

Les auteurs rapportent un tableau clinique atypique, celui d'un patient présentant un désordre du développement sexuel (46XY DDS). L'exploration a montré des taux de FSH et LH élevés, une testostéronémie basse, avec absence de gonades ainsi que tout dérivé des canaux de Müller et de Wolff à la laparoscopie.

Les auteurs discutent les différentes possibilités diagnostiques pouvant correspondre à ce tableau clinique et insistent sur celui de syndrome de régression testiculaire embryonnaire, entité rare et originale.

Mots clés : désordre du développement sexuel (46XY $D D S)$, régression testiculaire, hypoplasie des cellules de Leydig

\section{OBSERVATION}

La jeune BS, âgée de 16 ans, de morphotype féminin, consulte pour une aménorrhée primaire. Elle n'a pas d'antécédents pathologiques, est issue d'une grossesse normale et de parents sains non consanguins. Elle a deux frères et une soeur en bonne santé. II n'a pas été relevé d'antécédents familiaux particulier d'hypogonadisme chez des hommes, ou d'ectopie testiculaire.

L'examen physique montre un poids à $61 \mathrm{~kg}$, une taille à 1,54 $\mathrm{m}$, un développement statural normal, un index de masse corporelle à $25,7 \mathrm{~kg} / \mathrm{m} 2$, une absence de développement des seins et une absence de signes de virilisation. Le développement intellectuel était normal, en particulier il n'a pas été noté un retard scolaire.

L'examen des organes génitaux montre des grandes et petites lèvres de type infantile, sans ambiguïté, un hymen présent et perforé, le vagin est borgne et limité à un cul de sac de $2 \mathrm{~cm}$ de profondeur. II n'y a pas de pilosité axillaire et pubienne. Le toucher rectal ne trouve pas d'utérus palpable. Le reste de l'examen ne montre pas d'anomalie, en particulier l'auscultation cardiaque qui était normale ainsi que le labstix (pas de protéinurie). L'échographie abdominale montre deux reins d'échostructure et de topographie normale.

La biologie a montré des taux de FSH $(96,2 \mathrm{Ul} / \mathrm{ml})$ et $\mathrm{LH}(78 \mathrm{Ul} / \mathrm{ml})$ élevés et une testostéronémie très basse (à $0,13 \mathrm{mg} / \mathrm{l})$. La radiographie du poignet a montré un âge osseux inférieur à 13 ans (méthode de lecture comme pour les garçons). Le caryotype était de type $46, X Y$.

La laparoscopie n'a objectivé aucun dérivé des canaux de Muller et de Wolff, ni la présence de tissu gonadique, même au niveau du trajet des canaux inguinaux. Le test au Synacthène avec dosage du cortisol était normal. Après discussion avec la famille, la patiente est mise sous traitement hormonal substitutif par œstroprogestatifs.

\section{Correspondance :}

Dr Walid DENGUEZLI - 2 Avenue Jelloul Ben Chérifa, 4051 Sousse, Tunisie - $\mathrm{Tel}+21698698211$ -

Email walid.denguezli@ms.tn 


\section{COMMENTAIRES}

II s'agit d'un désordre de développement sexuel (46XY DDS) avec des organes génitaux externes féminins et un caryotype $46, X Y$. L'absence de gonades à la laparoscopie pourrait laisser supposer qu'il s'agit de reliquats fibreux observés en cas de dysgénésie gonadique.

Devant ce tableau clinique et biologique, le premier diagnostic qui pourrait se poser est celui de dysgénésie gonadique pure par une mutation du gène SRY. Cependant, ce diagnostic est peu probable devant l'absence d'utérus. Par ailleurs le reste des éléments cliniques de la patiente sans aucun développement mammaire, sans gonade palpable ou à la laparoscopie, la testostérone quasi indosable, éliminent une insensibilité par mutation des récepteurs aux androgènes (testicule féminisant). En effet, l'insensibilité aux androgènes est due à une mutation du gène du récepteur aux androgènes localisé en Xq11-q12 [13]. L'aspect des organes génitaux externes est variable allant du phénotype féminin au phénotype masculin avec micropénis [13].

Les taux de testostérone au cours de l'insensibilité aux androgènes sont élevés. Notre patiente a un taux effondré à $0,13 \mathrm{ng} / \mathrm{l}$ faisant éliminer ce diagnostic. Le diagnostic de bloc enzymatique sur la voie de synthèse de la testostérone repose en partie sur les résultats du test aux HCG. Au cours de ce test, l'évaluation des taux des précurseurs de la testostérone permet de diagnostiquer l'enzyme déficitaire. Les blocs enzymatiques qui restent très peu probables chez cette patiente sont le déficit en $3 \beta$ hydroxystéroidesdéshydrogénase, en StAR protéine, en 17- $ß$ hydroxystéroide déshydrogénase, et en $5 \alpha$-réductase. Les deux premiers s'accompagnent d'une insuffisance surrénalienne néonatale. Dans le troisième cas se produit une virilisation des organes génitaux externes en période pubertaire [16]. Enfin, dans le bloc en $5 \alpha-$ réductase les taux de testostérone sont normaux, ces éléments ne sont pas retrouvés chez notre patiente. II est de même pour le bloc en $17 \alpha$ hydroxylase, éliminé chez elle puisque le test au Synacthène avec dosage du cortisol est normal [8]. Reste donc à chercher des mutations génétiques autres que SRY intervenant dans le déterminisme sexuel.

En effet, une cascade de gènes impliqués dans la différenciation normale d'une gonade mâle a été décrite ces dernières années ; celle-ci se fait entre la 5 ème et la 8ème semaine de gestation. L'atteinte de chacun de ces gènes peut être responsable d'une dysgénésie gonadique [15]. Le premier gène décrit est le gène SRY (testis determining factor situé sur le bras court du chromosome $Y$ ) [14]. Cependant, les mutations du gène SRY n'expliquent que $20 \%$ des syndromes d'ambiguïté sexuelle.

D'autres gènes sont impliqués tel que WT1 (Wilms tumor gene 1) $[1,3,5,7,8,17]$, localisé sur le chromosome 11. Ses atteintes sont responsables de dysgénésie gonadique et d'atteinte rénale chez la plupart des individus 46,XY. Le gène SOX9 (SRY-related HMG box 9), est localisé sur le chromosome 17 [5, 6, 9, 11]. Son inactivation $[1,13]$ associe des anomalies osseuses diffuses et une ambiguïté sexuelle chez les sujets 46 , $X Y$. Le gène DAX (dosage sensitive sex reversal adrenal hypoplasia congenitale (AHC) critical region on the chromosome, gene 1) est localisé en Xp21 [1, 14]. Sa duplication chez un sujet $46, X Y$ entraîne une dysgénésie gonadique. Le gène SF1 (Stéroidogenic Factor 1) est localisé en $9 q$ [7]. II code pour un récepteur nucléaire régulant la transcription de protéines impliquées dans la stéroidogénèse surrénalienne et gonadique. SF1 régule également l'expression des hormones hypophysaires (sous-unité bêta de la LH et la FSH) [17]. La mutation de SF1 entraîne une agénésie complète des gonades et des glandes surrénales.

Malgré la découverte de tous ces gènes, la cause de la majorité des dysgénésies gonadiques reste inconnue à ce jour. Chez notre patiente, l'absence de dérivés Mullériens témoigne d'une sécrétion d'hormone antiMüllérienne $(A M H)$ par les cellules de Sertoli et fait éliminer le diagnostic de dysgénésie gonadique. Sous l'effet de l'AMH, la régression des dérivés Mullériens se fait dans le sens cranio-caudal $[8,10,12]$ et se déroule vers la 8 ème semaine de gestation, période où l'activité de cette hormone est maximale. Le diagnostic de dysgénésie gonadique étant éliminé chez cette patiente, nous devons discuter dans cette observation toutes les causes de pseudohermaphrodisme masculin avec absence de dérivés Mullériens.

L'hypoplasie des cellules de Leydig est due à une mutation inactivatrice du gène du récepteur de la $\mathrm{LH}$. Le nombre de patients rapportés dans la littérature n'excède pas 20 cas. L'inactivation peut être partielle ou complète et l'aspect des organes génitaux externes varie du masculin avec simple micropénis ou hypospadias, au féminin apparemment normal. En cas de phénotype féminin une absence de développement, mammaire est observée ainsi qu'une absence de pilosité axillaire et pubienne. Les dérivés Mullériens sont absents. Des dérivés wolffiens rudimentaires ont été observés chez certains patients en dépit de l'existence d'organes génitaux externes féminins [2].

Chez notre patiente il n'existe aucun développement des caractères sexuels secondaires, et les dérivés Wolffiens 
et Mullériens sont absents. Les taux de testostérone dans l'hypoplasie des cellules de Leydig sont bas et non stimulables par les HCG. Les taux de LH et de FSH sont élevés. Chez notre patiente, les taux de testostérone sont bas et les taux de FSH et LH sont élevés. Il existe des formes familiales de cette maladie ou une transmission autosomale récessive a été décrite. Aucun cas familial n'est rapporté dans la famille de notre patiente. Un test aux HCG est nécessaire chez notre patiente pour évaluer les capacités fonctionnelles de la cellule de Leydig. Si les taux de testostérone restent bas après stimulation, le diagnostic d'hypoplasie des cellules de Leydig pourra être évoqué puis confirmé par une étude en biologie moléculaire du gène du récepteur à la $\mathrm{LH}$. Enfin un diagnostic exceptionnel pourrait parfaitement coïncider avec ce tableau, il s'agit du syndrome de régression testiculaire embryonnaire.

Le syndrome de régression testiculaire $[6,9,11]$ se caractérise par l'association d'un caryotype $46, X Y$ et de testicules absents ou rudimentaires. L'étiopathogénie de ce syndrome n'est pas claire : l'involution testiculaire intra-utérine peut être due à une atteinte génétique, à un facteur tératogène ou à une torsion testiculaire. Le phénotype va de l'ambiguïté sexuelle sévère aux organes génitaux masculins avec micropénis [16].

Chez notre patiente, l'absence de gonades à la laparoscopie et d'utérus peut être expliquée par une ébauche de sécrétion d'AMH ayant engendré la régression des canaux de Müller. Cette étape a été probablement suivie par le phénomène causal ayant entraîné la disparition des gonades très précocement avant la 12ème semaine (stade embryonnaire) avant toute différenciation dans le sens masculin, ce qui pourrait expliquer le phénotype féminin. Seule la conjonction de ces deux phénomènes pourrait expliquer ce tableau clinique ; on arrive ainsi à situer le moment de survenue de la disparition des gonades entre la $8^{\text {ème }}$ et la $12^{\text {ème }}$ semaine. Bien qu'il soit rare ce diagnostic a été rapporté par certains auteurs dans des publications néanmoins anciennes [4, 6, 9]. Cependant, dans notre observation, il ne pourra être retenu qu'après avoir éliminé une mutation génétique WT1 ou SF1 et une hypoplasie des cellules de Leydig.

\section{CONCLUSION}

Le diagnostic étiologique des ambiguïtés sexuelles est difficile. L'approche en est multidisciplinaire. II impose la confrontation entre l'examen clinique, l'imagerie, l'hormonologie et la biologie moléculaire.

\section{REFERENCES}

1. ACHERMANN J.J., OZISIK G., MEEKS J.J., JAMESON J.L.: Genetic causes of human Reproductive disease. J. Clin. Endocrinol. Metab., 2002, 87 : 2447-2454.

2. AXELP., THEMMEN N., YERHOEF-POST M. : LH Receptor defect. Seminars in reproductive medicine. New York, Thieme, 2002, $20: 199-204$.

3. BOWLES., KOOPMAN P. : New Clues to the puzzle of mammalian sex determination. Genome Biol., 2001, 2 : 1025 : 1-4.

4. COULAM C.B. : Testicular regression syndrome. Obstet. Gynecol., 1979, $53:$ 44-49.

5. HOSSAIN A., SANDERS G. : The human sex-determining gene SRY is a direct target of Wt-1. J. Biol. Chem., 2001, 276: 16817-16823.

6. JOSSO N., BRIARD M.L. : Embryonic testicular regression syndrome : variable phenotypic expression in siblings. J. Pediatr., 1980, $97:$ 200-204.

7. KIM J., PRAVIT D., BARDEESY N. et al. : The Wilm's tumor suppressor gene (Wt1) product regulates Dax-1 gene expression during gonadal differentiation. Mol. Cell. Biol.,1999, $19: 2289-2299$.

8. MACLAUGHLIN D., DONAHOE P.K. : Sex determination and differentiation. N. Eng. J. Med., 350 : 367-368.

9. NAFFAH J. : Syndrome de régression testiculaire familial. Bull. Acad. Natl Med., 1989, 173 : 709-715.

10. NEF S., FARADAL. : Hormones in male sexual development. Genes Dev., 2000, 14 : 3075-3086.

11. OTTOLENGHI C., FILHO M., MENDONCAC. et al. : Absence of mutations involving the Lim home box domain gene $\mathrm{LHX} 9$ in 46,XY gonadal agenesis and dysgenesis. J. Clin. Endocrinol. Metab., 2001, $86:$ 2449-2465.

12. PIERSON M., VIDAILHET M., WILBERCQ L., PALANDRI Y.W., COMBESCURE B. : Le syndrome de régression testiculaire totale ou anorchidie. Arch. Fr. Pédiatr., 1983, 40: 467-473.

13. RAVEL C., CHANTOT-BASTARAUD S., SIFFOROI J.P. : Aspects moléculaires du déterminisme sexuel : régulation génique et pathologie. Gynecol. Obstet. Fertil., 2004, 32 : 584-594.

14. RAZAVIF E., ESCUDIER E. : Embryologie. Encycl. Méd. Chir. Gynécologie-Obstétrique, 2002, 5.001-a-50: 26.

15. RICHARD N.Y.I., ITO M., JAMESON J.L. : The murine DaxI promoter is stimulated by SF-1 (Steroidogenic Factor-1) and inhibited by COUP-TF(Chiken Ovalbumin Upstrearn Promoter-Transcription factor) via a composite nuclear receptor-Regulatory Element. Mol. Endocrinol., 1998, 12 : 1010-1022

16. SULTAN C., PARIS F., JANDEL C.: Ambiguous genitalia in the new born, normal and abnormal sexual differentiation : from genes to patient. Seminars in reproductive medicine. New York, Thieme, 2002, $20: 181-188$.

17. SWAIN A., BADGER R.L. : Mammalian sex determination. A molecular drama. Genes Dev., 1999, 13 : 755-767.

Manuscrit reçu : octobre 2006 ; accepté mars 2007. 


\begin{abstract}
Differential diagnoses of delayed puberty

Walid DENGUEZLI, Zina CHOUKET, Raja FALEH, Anis HADDAD,Zahra SAIDANI, Hayet LAAJILI, Mohamed SAKOUHI
\end{abstract}

The authors report a case of a 16-year-old Tunisian phenotypically female patient with an $46, X Y$ karyotype. Serum assays showed high serum FSH and LH levels and low serum testosterone levels. Laparoscopic exploration was performed to distinguish between testicular feminization syndrome and gonadal dysgenesis. No gonads and no persistent Müllerian or Wolffian ducts were found.

The authors suspected the diagnosis of embryonic testicular regression syndrome (TRS), a congenital condition in which the testes disappear during early embryonic development. A spectrum of pathological findings may be present, but few systematic descriptions have been reported in the literature.

The authors describe a new case of suspected embryonic testicular regression syndrome and discuss the differential diagnoses.

Key Words : pseudohermaphroditism, vanishing testis, Leydig cell hypoplasia 